\title{
Fractional Operators in $p$-adic Variable Exponent Lebesgue Spaces and Application to $p$-adic Derivative
}

\author{
Leonardo Fabio Chacón-Cortés $\mathbb{D D}^{1}$ and Humberto Rafeiro $\mathbb{D}^{2}$ \\ ${ }^{1}$ Departamento de Matemáticas, Facultad de Ciencias, Pontificia Universidad Javeriana, Cra. 7 No. 43-82, Bogotá, Colombia \\ ${ }^{2}$ Department of Mathematical Sciences, United Arab Emirates University, P.O. Box 15551, Al Ain, UAE
}

Correspondence should be addressed to Humberto Rafeiro; rafeiro@uaeu.ac.ae

Received 3 June 2021; Accepted 7 August 2021; Published 18 September 2021

Academic Editor: Douadi Drihem

Copyright (C) 2021 Leonardo Fabio Chacón-Cortés and Humberto Rafeiro. This is an open access article distributed under the Creative Commons Attribution License, which permits unrestricted use, distribution, and reproduction in any medium, provided the original work is properly cited.

In this paper, we prove the boundedness of the fractional maximal and the fractional integral operator in the $p$-adic variable exponent Lebesgue spaces. As an application, we show the existence and uniqueness of the solution for a nonhomogeneous Cauchy problem in the $p$-adic variable exponent Lebesgue spaces.

\section{Introduction}

The field of $p$-adic numbers are an interesting and useful tool to study phenomena in physics, biology, and medicine, among other sciences; see, e.g., [1-4] and references therein. For this reason, the study of operators that allows us to describe such phenomena is essential. Even more so when in the $p$-adic setting it is not possible to define the derivative in the classical sense.

Variable exponent Lebesgue spaces generalize the notion of $q$-integrability in the classical Lebesgue spaces, allowing the exponent to be a measurable function. These spaces were introduced in 1931 by Orlicz [5] but lay essentially dormant for more than 50 years. They received a thrust in the paper [6] and are now an active area of research having many known applications, e.g., in the modeling of thermorheological fluids [7] as well as electrorheological fluids [8-11], in differential equations with nonstandard growth $[12,13]$, and in the study of image processing [14-20]. For a thorough history, theory, and applications of variable exponent Lebesgue spaces, see $[6,21-24]$.

In this article, we are interested in the boundedness of the fractional integral and maximal fractional operator on the $p$-adic Lebesgue spaces with a variable exponent. The corresponding result for classical $p$-adic Lebesgue space is known (cf. [25]). These operators play an important role in such areas such as Sobolev spaces, potential theory, PDEs, and integral geometry, to name a few.
This work is divided as follows. Section 2 contains a quick description of the preliminary on the topic of the $p$-adic analysis and variable exponent Lebesgue spaces on the $p$-adic numbers, necessary for the development of this work. In Section 3, the boundedness of the fractional maximal operator

$$
M_{\alpha} f(x)=\sup _{\gamma \in \mathbb{Z}} \frac{1}{p^{\gamma(n-\alpha)}} \int_{B_{\gamma}^{n}(x)}|f(y)| d y, \quad x \in \mathbb{Q}_{p}^{n},
$$

is studied in the framework of variable exponent $p$-adic Lebesgue spaces. The boundedness of the special case $M_{0}$, the so-called Hardy-Littlewood maximal operator, was obtained in [26] under appropriate conditions on the exponent function. We prove, using a suitable pointwise estimate, the boundedness of the fractional maximal operator from $L^{q(\cdot)}\left(\mathbb{Q}_{p}^{n}\right)$ to $L^{q^{\#(\cdot)}}\left(\mathbb{Q}_{p}^{n}\right)$, where $q^{\#}$ is the Sobolev limiting exponent; see (31) for the corresponding definition. The boundedness of the fractional integral operator

$$
I_{\alpha} f(x)=\int_{\mathbb{Q}_{p}^{n}} \frac{f(y)}{\|x-y\|_{p}^{n-\alpha}} d y, \quad x \in \mathbb{Q}_{p}^{n},
$$

is obtained from the boundedness of the fractional maximal operator and Welland's pointwise inequality tailored for the 
p-adic setting; this approach is inspired from [27]. In the literature, it is customary to prove first the boundedness of the fractional potential operator and, as a corollary, the boundedness of the fractional maximal operator is obtained using the lattice property of the norm and the elementary estimate

$$
M_{\alpha} f(x) \leq I_{\alpha}|f|(x)
$$

As already mentioned, we will use a reverse approach.

Finally, in Section 4, we define the Taibleson operator in $p$-adic Lebesgue spaces with variable exponent, which is the analogue of the derivative in the spatial variable $x\left(x \in \mathbb{Q}_{p}^{n}\right)$, and study the nonhomogeneous Cauchy problem (72) associated with this operator.

The notation $a \lesssim b$ denotes the existence of a constant $C$ for which $a \leq C b, a \approx b$ means that $a \leqslant b$ and $b \leqq a$.

\section{Preliminaries}

For an exposition on the $p$-adic analysis, see $[25,28]$.

2.1. The Field of $p$-adic Numbers. By $p$ we denote a prime number. The field $\mathbb{Q}_{p}$ is given as the completion of $\mathbb{Q}$ with respect to the $p$-adic norm $|\cdot|_{p}$, given by

$$
|x|_{p}:= \begin{cases}0, & \text { if } x=0 \\ p^{-\gamma}, & \text { if } x=p^{\gamma} \frac{a}{b}\end{cases}
$$

where $a, b$ are integers coprime with $p$. The integer $\gamma:=$ ord $(x)(\operatorname{ord}(0):=+\infty)$ is denoted as the $p$-adic order of $x$. This norm can be extended to $\mathbb{Q}_{p}^{n}$ as

$$
\|x\|_{p}:=\max _{1 \leq i \leq n}\left|x_{i}\right|_{p} \quad \text { for } x=\left(x_{1}, \cdots, x_{n}\right) \in \mathbb{Q}_{p}^{n},
$$

and satisfies the so-called strong triangular inequality

$$
\|x+y\|_{p} \leq \max \left\{\|x\|_{p},\|y\|_{p}\right\}
$$

with equality when $\|x\|_{p} \neq\|y\|_{p}$. If $\operatorname{ord}(x):=\min _{1 \leq i \leq n}$ ord $\left.\left(x_{i}\right)\right\}$, it follows that $\|x\|_{p}=p^{-\operatorname{ord}(x)}$. The set $\left(\mathbb{Q}_{p}^{n},\|\cdot\|_{p}\right)$ is a complete ultrametric space and, as a topological space, $\mathbb{Q}_{p}$ is homeomorphic to a Cantor-like subset of the real line. A $p$-adic number $x \neq 0$ has a unique series expansion, viz.,

$$
x=p^{\operatorname{ord}(x)} \sum_{j=0}^{\infty} x_{j} p^{j}
$$

where $x_{j} \in\{0,1,2, \cdots, p-1\}$ and $x_{0} \neq 0$. For $\gamma \in \mathbb{Z}$, we denote by

$$
B_{\gamma}^{n}(a)=\left\{x \in \mathbb{Q}_{p}^{n}:\|x-a\|_{p} \leq p^{\gamma}\right\}
$$

the ball of radius $p^{\gamma}$ with center at $a=\left(a_{1}, \cdots, a_{n}\right) \in \mathbb{Q}_{p}^{n}$ and by

$$
S_{\gamma}^{n}(a)=\left\{x \in \mathbb{Q}_{p}^{n}:\|x-a\|_{p}=p^{\gamma}\right\}=B_{\gamma}^{n}(a) \backslash B_{\gamma-1}^{n}(a),
$$

the corresponding sphere. We denote

$$
B_{\gamma}^{n}(0):=B_{\gamma}^{n}, \quad S_{\gamma}^{n}(0):=S_{\gamma}^{n},
$$

and note that

$$
\mathbb{Q}_{p}^{n} \backslash\{0\}=\bigcup_{\gamma \in \mathbb{Z}} S_{\gamma}^{n}
$$

Note that $B_{\gamma}^{n}(a)=B_{\gamma}\left(a_{1}\right) \times \cdots \times B_{\gamma}\left(a_{n}\right)$, where $B_{\gamma}\left(a_{i}\right)$ $:=\left\{x \in \mathbb{Q}_{p}:\left|x-a_{i}\right|_{p} \leq p^{\gamma}\right\}$ is the one-dimensional ball. In $\mathbb{Q}_{p}^{n}$ there exists the additive Haar measure $d^{n} x:=d x$ (by $E \mid$ we denote the Haar measure of the set $E$ ), since the field $\mathbb{Q}_{p}^{n}$ is a locally compact commutative group with respect to addition. Normalizing the measure $d x$ by $\int_{B_{0}^{n}} d$ $x=1$, we get a unique measure. From here onwards, we use the normalized Haar measure; thus,

$$
\operatorname{Vol}\left(B_{\gamma}^{n}(a)\right)=p^{\gamma n}, \quad \operatorname{Vol}\left(S_{\gamma}^{n}(a)\right)=p^{\gamma n}\left(1-p^{-n}\right)
$$

for any $a \in \mathbb{Q}_{p}^{n}$.

Note that the collection of all disjoint balls of the same radius $\gamma$ forms a partition of $\mathbb{Q}_{p}^{n}$, since inequality (6) implies that any two balls in $\mathbb{Q}_{p}^{n}$ with the same radius are either identical or disjoint.

2.2. Some Function Spaces. A complex-valued function $\varphi$ defined on $\mathbb{Q}_{p}^{n}$ is called locally constant if for any $x \in \mathbb{Q}_{p}^{n}$, there exists an integer $l(x) \in \mathbb{Z}$ such that

$$
\varphi\left(x+x^{\prime}\right)=\varphi(x) \quad \text { for } x^{\prime} \in B_{l(x)}^{n} .
$$

A function $\varphi: \mathbb{Q}_{p}^{n} \longrightarrow \mathbb{C}$ is called a Schwartz-Bruhat function (or a test function) if it is locally constant with the compact support. The $\mathbb{C}$-vector space of SchwartzBruhat functions is denoted by $S\left(\mathbb{Q}_{p}^{n}\right):=S$.

A measurable function $f: \mathbb{Q}_{p}^{n} \longrightarrow \mathbb{C}$ belongs to the Lebesgue space $L^{q}\left(\mathbb{Q}_{p}^{n}\right), 1 \leq q<\infty$, when

$$
\|f\|_{L^{q}\left(\mathbb{Q}_{p}^{n}\right)}^{q}:=\int_{\mathbb{Q}_{p}^{n}}|f(x)|^{q} d x<\infty, \quad 1 \leq q<\infty,
$$

where

$$
\int_{\mathbb{Q}_{p}^{n}}|f(x)|^{q} d x:=\lim _{\gamma \longrightarrow \infty} \int_{B_{\gamma}^{n}(0)}|f(x)|^{q} d x,
$$

if the limit exists. 
We now introduce the notion of $p$-adic Lebesgue spaces with a variable exponent and give some properties needed in the sequel; see [26] for the respective proofs.

We say that a measurable function is a variable exponent if $q: \mathbb{Q}_{p}^{n} \longrightarrow[1, \infty)$. By $\mathbb{Q}\left(\mathbb{Q}_{p}^{n}\right)$ we denote the set of all vari-

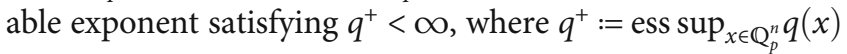
and $q^{-}:=\operatorname{essinf}_{x \in \mathbb{Q}_{p}^{n}} q(x)$.

For $q \in \mathbb{Q}\left(\mathbb{Q}_{p}^{n}\right)$ by $L^{q(\cdot)}\left(\mathbb{Q}_{p}^{n}\right)$, we denote the space of measurable functions $f: \mathbb{Q}_{p}^{n} \longrightarrow \mathbb{R}$ such that

$$
\|f\|_{L^{q(\cdot)}\left(\mathbb{Q}_{p}^{n}\right)}=\inf \left\{\lambda>0: \mathrm{Q}_{q(\cdot)}\left(\frac{f}{\lambda}\right) \leq 1\right\}<\infty
$$

where $\mathrm{\varrho}_{q(\cdot)}(f):=\int_{\mathbb{Q}_{p}^{n}}|f(x)|^{q(x)} d x$.

For the Lebesgue space with a variable exponent, we have

$$
\begin{aligned}
\|f\|_{L^{q(\cdot)}\left(\mathbb{Q}_{p}^{n}\right)} & \leq \mathrm{Q}_{q(\cdot)}(f)+1, \\
\mathrm{Q}_{q(\cdot)}(f) & \left.\leq\left(1+\|f\|_{L^{q(\cdot)}\left(\mathbb{Q}_{p}^{n}\right)}\right)\right)^{q^{+}}, \\
\|f\|_{L^{q(\cdot)}\left(\mathbb{Q}_{p}^{n}\right)} & =\left\||f|^{s}\right\|_{L^{(q) / s}\left(\mathbb{Q}_{p}^{n}\right)}^{1 / s}, \quad s \in\left(0, q^{-}\right] .
\end{aligned}
$$

The Hölder inequality is valid, up to a multiplicative constant, in the framework of Lebesgue spaces with variable exponent, viz.,

$$
\int_{\mathbb{Q}_{p}^{n}}|f(x) g(x)| d x \leq C\|f\|_{L^{q(\cdot)}\left(\mathbb{Q}_{p}^{n}\right)}\|g\|_{L^{q^{\prime}(\cdot)}\left(\mathbb{Q}_{p}^{n}\right)}
$$

where $q$ and $q^{\prime}$ are conjugate exponents, viz., $1=1 / q(x)+$ $1 / q^{\prime}(x)$.

For $q \in \mathbb{Q}\left(\mathbb{Q}_{p}^{n}\right)$, we say that $q \in W_{0}\left(\mathbb{Q}_{p}^{n}\right)$ when there is a positive constant $C$, for which

$$
\gamma\left(q^{-}\left(B_{\gamma}^{n}(x)\right)-q^{+}\left(B_{\gamma}^{n}(x)\right)\right) \leq C,
$$

for all $\gamma \in \mathbb{Z}$ and any $x \in \mathbb{Q}_{p}^{n}$. We say that $q \in W^{\infty}\left(\mathbb{Q}_{p}^{n}\right)$ when there is a positive constant $C$, for which

$$
|q(x)-q(y)| \leq C \frac{1}{\log _{p}\left(p+\min \left\{\|y\|_{p},\|x\|_{p}\right\}\right)},
$$

for any $x, y \in \mathbb{Q}_{p}^{n}$.

The class $W_{0}^{\infty}\left(\mathbb{Q}_{p}^{n}\right)$ is defined as $W_{0}^{\infty}\left(\mathbb{Q}_{p}^{n}\right):=W_{0}\left(\mathbb{Q}_{p}^{n}\right)$ $\cap W^{\infty}\left(\mathbb{Q}_{p}^{n}\right)$. The importance of the class $W_{0}^{\infty}\left(\mathbb{Q}_{p}^{n}\right)$ stems from the fact that it is a sufficient condition for the boundedness of the maximal operator in $L^{q(\cdot)}\left(\mathbb{Q}_{p}^{n}\right)$ : if $q \in W_{0}^{\infty}\left(\mathbb{Q}_{p}^{n}\right)$, then

$$
\|M f\|_{L^{q(\cdot)}\left(\mathbb{Q}_{p}^{n}\right)} \leq C\|f\|_{L^{q(\cdot)}\left(\mathbb{Q}_{p}^{n}\right)},
$$

see Theorem 5.2 of [26] for the corresponding proof.
In the case where $\Omega_{p}^{n}$ is a bounded subset of $\mathbb{Q}_{p}^{n}$, we have the following: if $q \in W_{0}\left(\mathbb{Q}_{p}^{n}\right)$, then,

$$
\|M f\|_{L^{q(\cdot)}\left(\Omega_{p}^{n}\right)} \leq C\|f\|_{L^{q(\cdot)}\left(\Omega_{p}^{n}\right)},
$$

see Theorem 5.1 of [26].

Lemma 1. Let $q \in \mathbb{Q}\left(\mathbb{Q}_{p}^{n}\right)$ be a L-Lipschitz function, for some $L \geq 0$. Then, $q \in W_{0}\left(\mathbb{Q}_{p}^{n}\right)$.

Proof. We give the proof only for the case $\gamma \leq 0$ since the other case is immediate. Since $q$ is a continuous function, for any ball $B_{\gamma}^{n}(x) \subset \mathbb{Q}_{p}^{n}$, there exists a maximum (respectively, minimum) point $\bar{x} \in B_{\gamma}^{n}(x)$ (respectively, $\underline{x} \in B_{\gamma}^{n}(x)$ ). From the Lipschitzianity of $q$, we have

$$
\begin{aligned}
& -\gamma\left[q^{+}\left(B_{\gamma}^{n}(x)\right)-q^{-}\left(B_{\gamma}^{n}(x)\right)\right]=-\gamma(q(\bar{x})-q(\underline{x})) \\
& \quad \leq-L \gamma\|\bar{x}-\underline{x}\|_{p} \leq 2 L \frac{|\gamma|}{p^{|\gamma|}} \leq C
\end{aligned}
$$

which completes the proof.

We now show an extension result via the well-known McShane extension technique (a similar approach was used in the Euclidean framework; see [23]).

Lemma 2. Let $q \in W_{0}\left(\Omega_{p}^{n}\right)$, where $\Omega_{p}^{n}$ is a bounded subset of $\mathbb{Q}_{p}^{n}$. Then, there exists an extension function $\breve{q} \in W_{0}^{\infty}\left(\mathbb{Q}_{p}^{n}\right)$ which is constant outside some fixed ball.

Proof. The proof will be divided into two steps.

First step: we show that there exists an extension function $\tilde{q} \in W_{0}\left(\mathbb{Q}_{p}^{n}\right)$. Let us define $\tilde{q}$ as

$$
\tilde{q}(x):=\sup _{\xi \in \Omega_{p}^{n}}\left[q(\xi)-\omega\left(\|x-\xi\|_{p}\right)\right]
$$

with

$$
\omega(t):= \begin{cases}\frac{C}{\log _{p}(p+1 / t)}, & t>0, \\ 0, & t=0,\end{cases}
$$

where $C$ comes from equation (21). Since $\omega(t)$ is an increasing and concave function for $t \geq 0$ and approaches zero with $t$, then from Theorem 2 of [29], we have that $|\tilde{q}(x)-\tilde{q}(y)|$ $\leq \omega\left(\|x-y\|_{p}\right)$. In order to prove that $\tilde{q} \in W_{0}\left(\mathbb{Q}_{p}^{n}\right)$, it suffices to check for $\gamma<0$. Since $\omega$ is an increasing function and taking $\bar{x}$ and $\underline{x}$ as in the proof of Lemma 1, we see that 


$$
\begin{aligned}
& \gamma\left(\tilde{q}^{-}\left(B_{\gamma}^{n}(x)\right)-\tilde{q}^{+}\left(B_{\gamma}^{n}(x)\right)\right)=\gamma(\tilde{q}(\underline{x})-\tilde{q}(\bar{x})) \\
& \quad=|\gamma \| \tilde{q}(\bar{x})-\tilde{q}(\underline{x})| \leq|\gamma| \omega\left(\|\bar{x}-\underline{x}\|_{p}\right) \\
& \quad \leq C \frac{|\gamma|}{\log _{p}\left(p+p^{|\gamma|}\right)} \leq C,
\end{aligned}
$$

which ends the first step.

Second step: we show that there exists an extension function $\breve{q} \in W_{0}^{\infty}\left(\mathbb{Q}_{p}^{n}\right)$. Since $\Omega_{p}^{n}$ is a bounded set, let us take $\gamma>0$ such that $\Omega_{p}^{n} \subset B_{\gamma}^{n}(0)$. We define two Urysohn functions, $u_{0}$ and $u_{\infty}$, as follows:

$$
\begin{aligned}
u_{0}(x) & =\frac{d\left(x, \mathbb{Q}_{p}^{n} \backslash B_{\gamma+1}^{n}(0)\right)}{d\left(x, \mathbb{Q}_{p}^{n} \backslash B_{\gamma+1}^{n}(0)\right)+d\left(x, \Omega_{p}^{n}\right)}, u_{\infty}(x) \\
& =\frac{d\left(x, \Omega_{p}^{n}\right)}{d\left(x, \mathbb{Q}_{p}^{n} \backslash B_{\gamma+1}^{n}(0)\right)+d\left(x, \Omega_{p}^{n}\right)} .
\end{aligned}
$$

The functions $u_{0}$ and $u_{\infty}$ are $L$-Lipschitz with $L \leq 1 / p$, due to the fact that $d\left(\Omega_{p}^{n}, \mathbb{Q}_{p}^{n} \backslash B_{\gamma+1}^{n}(0)\right) \geq p$, see Prop. 2.1.1 of [30]. Defining the exponent $\breve{q}$ as,

$$
\breve{q}(x):=\tilde{q}(x) u_{0}(x)+q^{+} u_{\infty}(x),
$$

we see that $\breve{q} \in W_{0}\left(\mathbb{Q}_{p}^{n}\right)$ since the class $W_{0}\left(\mathbb{Q}_{p}^{n}\right)$ is closed under addition and multiplication, and $\breve{q} \in W^{\infty}\left(\mathbb{Q}_{p}^{n}\right)$ because $\breve{q}(x) \equiv q^{+}$in the exterior of the ball $B_{\gamma+1}^{n}(0)$.

\section{Main Results}

3.1. Boundedness in $\mathbb{Q}_{p}^{n}$. In this section, we study the boundedness of the operators in the case of $\mathbb{Q}_{p}^{n}$.

3.1.1. Fractional Maximal Operator. The classical result regarding boundedness of the fractional maximal operator says that if $1<q<n / \alpha$ and $1 / s=1 / q-\alpha / n$, then $M_{\alpha}: L^{q}$ $\left(\mathbb{Q}_{p}^{n}\right) \longrightarrow L^{s}\left(\mathbb{Q}_{p}^{n}\right)$ is bounded (this follows at once from inequality (3) and the boundedness of the operator $I_{\alpha}: L^{q}$ $\left(\mathbb{Q}_{p}^{n}\right) \longrightarrow L^{s}\left(\mathbb{Q}_{p}^{n}\right)$, cf. [25]). For further goals, we need estimate (32).

Lemma 3. Let $0<\alpha<n, q$ be an exponent function such that $1<q^{-} \leq q^{+}<n / \alpha$, and we define the Sobolev limiting exponent $q^{\#}$ by

$$
\frac{1}{q^{\#}(x)}:=\frac{1}{q(x)}-\frac{\alpha}{n}
$$

Then,

$M_{\alpha} f(x) \leq c\left[M\left(|f(\cdot)|^{\left(q(\cdot) / q^{\sharp} \cdot(\cdot)\right)(n /(n-\alpha))}\right)(x)\right]^{1-\alpha / n}\left(\int_{\mathbb{Q}_{p}^{n}}|f(y)|^{q(y)} d y\right)^{\alpha / n}$.
Proof. From $q(x) / q^{\#}(x)+\alpha q(x) / n=1$ and Hölder's inequality (20), we have

$$
\begin{aligned}
\frac{1}{p^{p n-\gamma \alpha}} \int_{B_{\gamma}^{n}(x)}|f(y)| d y= & \frac{1}{p^{\gamma n-\gamma \alpha}} \int_{B_{\gamma}^{n}(x)}|f(y)|^{q(y) / q^{\#}(y)}|f(y)|^{\alpha q(y) / n} d y \\
\leq & c\left[\frac{1}{p^{\gamma-\gamma \alpha}} \int_{B_{\gamma}^{n}(x)}|f(y)|^{n q(y))\left[q^{\#}(y)(\alpha-n)\right]} d y\right]^{1-\alpha / n} \\
& \cdot\left(\int_{B_{\gamma}^{n}(x)}|f(y)|^{q(y)} d y\right)^{\alpha / n} .
\end{aligned}
$$

Taking the supremum over all $\gamma \in \mathbb{Z}$, we establish the desired inequality.

Theorem 4. Let $0<\alpha<n$ and $q$ be an exponent function such that $1<q^{-} \leq q^{+}<n / \alpha$. Suppose, moreover, that $q^{\#} \in W_{0}^{\infty}$ $\left(\mathbb{Q}_{p}^{n}\right)$, where $q^{\#}$ is the Sobolev limiting exponent (31). Then, the operator $M_{\alpha}: L^{q(\cdot)}\left(\mathbb{Q}_{p}^{n}\right) \longrightarrow L^{q^{\#}(\cdot)}\left(\mathbb{Q}_{p}^{n}\right)$ is bounded.

Proof. Taking $\|f\|_{L^{q(\cdot)}\left(\mathbb{Q}_{p}^{n}\right)}=1$, the estimate (32), the relation (19), and the boundedness of the maximal operator $M$ in $L^{q^{\#}(\cdot)}\left(\mathbb{Q}_{p}^{n}\right)$, we have

$$
\begin{aligned}
& \left\|M_{\alpha} f(x)\right\|_{L^{q^{\sharp} \cdot(\cdot)}\left(\mathbb{Q}_{p}^{n}\right)} \leqslant\left\|\left[M\left(|f(\cdot)|^{\left(q(\cdot) / q^{\#}(\cdot)\right)(n /(n-\alpha))}\right)(x)\right]^{1-\alpha / n}\right\|_{L^{q^{*}(\cdot)}\left(\mathbb{Q}_{p}^{n}\right)} \\
& =\left\|M\left(|f(\cdot)|^{\left(q(\cdot) / q^{\sharp}(\cdot)\right)(n /(n-\alpha))}\right)(x)\right\|_{L^{4^{\sharp}(\cdot)(1-\alpha / n)}\left(\mathbb{Q}_{p}^{n}\right)}^{1-\alpha / n} \\
& \lesssim_{\alpha, n, p}\left\|\left.|f(\cdot)|\right|^{\left.(q \cdot \cdot) / q^{\#}(\cdot)\right)(n /(n-\alpha))}\right\|_{L^{q^{*}(\cdot)(1-\alpha / n)}\left(\mathbb{Q}_{p}^{n}\right)}^{1-\alpha / n} \\
& \lesssim_{\alpha, n, p}\left\||f(\cdot)|^{q(\cdot) / q^{\#}(\cdot)}\right\|_{L^{q^{\sharp}(\cdot)}\left(\mathbb{Q}_{p}^{n}\right)} \lessgtr_{\alpha, n, p} 1 .
\end{aligned}
$$

The general case follows from homogeneity considerations.

3.1.2. Fractional Potential Operator. The well-known Sobolev theorem states that the fractional potential operator (2), sometimes introduced with a normalizing factor, is bounded from $L^{q}\left(\mathbb{Q}_{p}^{n}\right)$ to $L^{q^{\#}}\left(\mathbb{Q}_{p}^{n}\right)$ where $1 / q^{\#}=1 / q-\alpha / n$ is the socalled Sobolev limiting exponent; see, for instance, [25].

In order to obtain the boundedness result in the variable exponent framework, we first obtain the validity of a Welland-type estimate in the $p$-adic setting; see [31] for the Euclidean counterpart.

Lemma 5. Let $0<\alpha<n, 0<\varepsilon<\max (\alpha, n-\alpha)$, and $f \in L_{\text {loc }}^{l}$ $\left(\mathbb{Q}_{p}^{n}\right)$. Then,

$$
\left|I_{\alpha} f(x)\right| \lesssim_{\alpha, n, p}\left|M_{\alpha-\varepsilon} f(x)\right|^{1 / 2}\left|M_{\alpha+\varepsilon} f(x)\right|^{1 / 2} .
$$


Proof. Let $\gamma \in \mathbb{Z}$, then

$$
\begin{aligned}
\int_{B_{\gamma}^{n}(x)} \frac{|f(y)|}{\|x-y\|_{p}^{n-\alpha} d y} & =\sum_{k=-\infty}^{\gamma} \frac{1}{p^{k(n-\alpha)}} \int_{S_{k}^{n}(x)}|f(y)| d y \\
& \leq \sum_{k=-\infty}^{\gamma} p^{k \varepsilon} \frac{1}{p^{k(n-\alpha+\varepsilon)}} \int_{B_{k}^{n}(x)}|f(y)| d y \\
& \leq \sum_{k=-\infty}^{\gamma} p^{k \varepsilon} M_{\alpha-\varepsilon} f(x) \lesssim_{\alpha, n, p} \frac{p^{\varepsilon(\gamma+1)}}{1-p^{-\varepsilon}} M_{\alpha-\varepsilon} f(x) \\
& \lesssim_{\alpha, n, p, \varepsilon} p^{\varepsilon \gamma} M_{\alpha-\varepsilon} f(x) .
\end{aligned}
$$

On the other hand,

$$
\begin{aligned}
\int_{C_{B_{\gamma}^{n}(x)} \frac{|f(y)|}{\|x-y\|_{p}^{n-\alpha}} d y} & =\sum_{k=\gamma+1}^{\infty} \frac{1}{p^{k(n-\alpha)}} \int_{S_{k}^{n}(x)}|f(y)| d y \\
& \leq \sum_{k=\gamma+1}^{\infty} p^{-k \varepsilon} \frac{1}{p^{k(n-\alpha-\varepsilon)}} \int_{B_{k}^{n}(x)}|f(y)| d y \\
& \leq \sum_{k=\gamma+1}^{\infty} p^{-k \varepsilon} M_{\alpha+\varepsilon} f(x) \lesssim_{\alpha, n, p} \frac{p^{\varepsilon(-\gamma+1)}}{1-p^{-\varepsilon}} M_{\alpha+\varepsilon} f(x) \\
& \lesssim_{\alpha, n, p} \frac{p^{\varepsilon(-\gamma+1)}}{1-p^{-\varepsilon}} M_{\alpha+\varepsilon} f(x) \lessgtr_{\alpha, n, p} p^{-\varepsilon \gamma} M_{\alpha+\varepsilon} f(x) .
\end{aligned}
$$

Taking the previous estimates into consideration, we have

$$
\left|I_{\alpha} f(x)\right| \lesssim_{\alpha, n, p, \varepsilon} p^{\varepsilon \gamma} M_{\alpha-\varepsilon} f(x)+p^{-\varepsilon \gamma} M_{\alpha+\varepsilon} f(x) .
$$

The inequality (35) is obtained taking into account (38) with $\gamma$ given by

$$
\gamma=\left\lceil\frac{\log _{p}\left[M_{\alpha+\varepsilon} f(x) / M_{\alpha-\varepsilon} f(x)\right]^{1 / 2}}{\varepsilon}\right\rceil,
$$

since $x \leq\lceil x\rceil<x+1$.

Theorem 6. Let $1<q^{-} \leq q^{+} \leq n / \alpha$ and $q \in W_{0}^{\infty}\left(\mathbb{Q}_{p}^{n}\right)$. Then, the fractional potential operator,

$$
I_{\alpha}: L^{q(\cdot)}\left(\mathbb{Q}_{p}^{n}\right) \longrightarrow L^{q^{\#}(\cdot)}\left(\mathbb{Q}_{p}^{n}\right)
$$

is bounded, where $q^{\#}$ is the Sobolev limiting exponent (31).

Proof. From the definition of the variable exponent Lebesgue norm and homogeneity, it suffices to show that

$$
\int_{\mathbb{Q}_{p}^{n}}\left|I_{\alpha} f(x)\right|^{q^{\#}(x)} d x \leq C,
$$

From (35) and Hölder's inequality, we obtain

$$
\begin{aligned}
\int_{\mathbb{Q}_{p}^{n}}\left|I_{\alpha} f(x)\right|^{q^{\#}(x)} d x & \lesssim_{\alpha, n, p} \int_{\mathbb{Q}_{p}^{n}}\left|M_{\alpha-\varepsilon} f(x)\right|^{q^{\#}(x) / 2}\left|M_{\alpha+\varepsilon} f(x)\right|^{q^{\#}(x) / 2} d x \\
& \lesssim_{\alpha, n, p}\left\|\left(M_{\alpha-\varepsilon} f(\cdot)\right)^{q^{\#}(\cdot) / 2}\right\|_{q_{1}(\cdot)}\left\|\left(M_{\alpha+\varepsilon} f(\cdot)\right)^{q^{\#}(\cdot) / 2}\right\|_{q_{2}(\cdot)} \\
& \leq c I_{1} I_{2},
\end{aligned}
$$

where $q_{1}$ and $q_{2}$ are conjugate exponents and will be chosen appropriately. To estimate $I_{1}$, from (17), we have

$$
\begin{aligned}
I_{1} & \leq \mathrm{Q}_{q_{1}(\cdot)}\left(\left(M_{\alpha-\varepsilon} f(\cdot)\right)^{q^{\#}(\cdot) / 2}\right)+1 \\
& =\int_{\mathbb{Q}_{p}^{n}}\left(M_{\alpha-\varepsilon} f(x)\right)^{q^{\#}(x) q_{1}(x) / 2} d x+1 .
\end{aligned}
$$

Defining $q_{1}$ by $1 / q(x)-2 /\left[q^{\#}(x) q_{1}(x)\right]=(\alpha-\varepsilon) / n$, we have, by Lemma 3 , that the operator $M_{\alpha-\varepsilon}: L^{q(\cdot)}\left(\mathbb{Q}_{p}^{n}\right) \longrightarrow$ $L^{\tilde{q}(\cdot)}\left(\mathbb{Q}_{p}^{n}\right)$ is bounded, where $\tilde{q}(x)=q^{\#}(x) q_{1}(x) / 2$. From (43) and (18), it follows that

$$
\begin{aligned}
\left\|\left(M_{\alpha-\varepsilon} f(\cdot)\right)^{q^{\#}(\cdot) / 2}\right\|_{q_{1}(\cdot)} & \leq\left(1+\left\|M_{\alpha-\varepsilon} f\right\|_{L^{\tilde{q}(\cdot)}\left(\mathbb{Q}_{p}^{n}\right)}\right)^{\tilde{q}^{+}}+1 \\
& \leq c\left(1+\|f\|_{L^{q(\cdot)}\left(\mathbb{Q}_{p}^{n}\right)}\right)^{\tilde{q}^{+}}+1 \leq c,
\end{aligned}
$$

which end the estimate for $I_{1}$.

The estimate for $I_{2}$ follows, mutatis mutandis, as $I_{1}$ taking into account that defining $q_{2}$ as $1 / q(x)-2 /\left[q^{\#}(x) q_{2}(x)\right]$ $=(\alpha+\varepsilon) / n$, we get that $q_{1}$ and $q_{2}$ are indeed conjugate exponents and $q_{2}$ is the right exponent for the boundedness of $M_{\alpha+\varepsilon}$; the details are left to the reader.

3.2. Boundedness in $\Omega_{p}^{n}$. The fractional integral operator $I_{\alpha}$ can be defined for an open set $\Omega_{p}^{n} \subset \mathbb{Q}_{p}^{n}$ in the following way:

$$
I_{\alpha} f(x)=\int_{\Omega_{p}^{n}} \frac{f(y)}{\|x-y\|_{p}^{n-\alpha}} d y, \quad x \in \Omega_{p}^{n} .
$$

We are interested in proving the $L^{q(\cdot)}\left(\Omega_{p}^{n}\right) \longrightarrow L^{q^{\#}(\cdot)}\left(\Omega_{p}^{n}\right)$ boundedness for the operator $I_{\alpha}$, where $q^{\#}$ is defined by (31). We begin with two lemmas, which are important on their own.

Although we need Lemma 7 for bounded set $\Omega_{p}^{n}$, we give the lemma for general measurable sets $\Omega_{p}^{n}$ which include, as a particular case, $\Omega_{p}^{n}=\mathbb{Q}_{p}^{n}$.

Lemma 7. Let $q \in W_{0}^{\infty}\left(\Omega_{p}^{n}\right)$. Then,

$$
\left\|\chi_{B_{\gamma}^{n}(x)}(\cdot)\right\|_{L^{q(\cdot)}\left(\Omega_{p}^{n}\right)} \leq C p^{\gamma n / q(x, \gamma)},
$$




$$
q(x, \gamma)= \begin{cases}q(x), & \gamma<0 \\ q(\infty), & \gamma \geq 0\end{cases}
$$

Proof. We split the proof into three cases: (1) $\gamma<0$, (2) $\gamma \geq 0$ and $p^{\gamma} \leq\|x\|_{p}$, and (3) $\gamma \geq 0$ and $p^{\gamma}>\|x\|_{p}$.

Case 1. $\gamma<0$. Since $q \in W_{0}\left(\Omega_{p}^{n}\right)$, we see that

$$
p^{\gamma n(q(y) / q(x)-1)}=p^{\gamma n((q(y)-q(x)) / q(x))} \geq p^{\gamma n\left(\left(q^{+}-q^{-}\right) / q^{-}\right)} \geq p^{-C},
$$

thus, $p^{-\gamma n(q(y) / q(x))} \leq p^{-\gamma n} p^{C}$. Integrating the last inequality over $B_{\gamma}^{n}(x)$, we obtain

$$
\int_{B_{\gamma}^{n}(x)} \frac{1}{p^{\gamma n(q(y) / q(x))}} d y \leq \int_{B_{\gamma}^{n}(x)} p^{-\gamma n} p^{C} d y \leq p^{C},
$$

from which it follows, using the definition of variable exponent norm and (49), that

$$
\left\|\chi_{B_{\gamma}^{n}(x)}(\cdot)\right\|_{L^{q(\cdot)}\left(\mathbb{Q}_{p}^{n}\right)} \lesssim p^{\gamma n / q(x)} .
$$

Case 2. $\gamma \geq 0$ and $p^{\gamma} \leq\|x\|_{p}$. In this case, we have $\|y\|_{p} \geq$ $\|x\|_{p}-\|x-y\|_{p} \geq p^{\gamma}(p-1)$ (because $\left.y \in B_{\gamma}^{n}(x)\right)$. Since $q \in$ $W^{\infty}\left(\mathbb{Q}_{p}^{n}\right)$, then taking limit when $\|x\|_{p} \longrightarrow \infty$, we get

$$
|q(\infty)-q(y)| \leq \frac{C}{\log _{p}\left(p+\|y\|_{p}\right)}
$$

from which we obtain

$p^{\gamma n(1-q(y) / q(\infty))}=p^{\gamma n((q(\infty)-q(y)) / q(\infty))} \leq p^{\gamma n\left(C / \log _{p}\left(p+\|y\|_{p}\right)\right)} \leq p^{C}$,

consequently, $p^{-\gamma n(q(y) / q(\infty))} \leq p^{-\gamma n+C}$. Integrating the last inequality over $B_{\gamma}^{n}(x)$, we have

$$
\int_{B_{\gamma}^{n}(x)} \frac{1}{p^{\gamma n(q(y) / q(\infty))}} d y \leq \int_{B_{\gamma}^{n}(x)} p^{-\gamma n+C} d y \leq p^{C},
$$

from which, by the definition of variable exponent norm, we get

$$
\left\|\chi_{B_{\gamma}^{n}(x)}(\cdot)\right\|_{L^{q(\cdot)}\left(\mathbb{Q}_{p}^{n}\right)} \lesssim p^{\gamma n / q(\infty)}
$$

Case 3. $\gamma \geq 0$ and $\|x\|_{p}<p^{\gamma}$. By the ultrametricity and the condition on $\|x\|_{p}$, we have $B_{\gamma}^{n}(x) \subset B_{\gamma}^{n}(0)$. Then,

$$
\begin{aligned}
\int_{B_{\gamma}^{n}(x)} \frac{1}{p^{\gamma n(q(y) / q(\infty))}} d y & \leq \int_{B_{\gamma}^{n}(0)} \frac{1}{p^{\gamma n(q(y) / q(\infty))}} d y \\
& \lesssim \int_{B_{\gamma}^{n}(0)} \frac{1}{p^{\gamma^{n}}} d y \lesssim \frac{1}{p^{\gamma n}} \int_{B_{\gamma}^{n}(0)} d y \lesssim 1,
\end{aligned}
$$

from which, by the definition of variable exponent norm, we get

$$
\left\|\chi_{B_{\gamma}^{n}(x)}(\cdot)\right\|_{L^{q(\cdot)}\left(\mathbb{Q}_{p}^{n}\right)} \lesssim p^{\gamma n / q(\infty)},
$$

which completes the proof.

Lemma 8. Let $q \in W_{0}^{\infty}\left(\mathbb{Q}_{p}^{n}\right)$. Then,

$$
\left\|\frac{\chi_{\mathbb{Q}_{p}^{n} \backslash B_{\gamma}^{n}(x)}(\cdot)}{\|x-\cdot\|_{p}^{\beta}}\right\|_{L^{q(\cdot)}\left(\mathbb{Q}_{p}^{n}\right)} \leq C p^{\gamma n / q(x, \gamma)-\gamma \beta},
$$

where $q(x, \gamma)$ is defined in (47).

Proof. We first prove the theorem for the case $\beta=n$. When $y \notin B_{\gamma}^{n}(x)$, by the ultrametricity condition, we have $B_{\gamma}^{n}(x) \subset$ $B_{-\operatorname{ord}(x-y)}^{n}(y)$; thus,

$$
\begin{aligned}
M\left(\frac{\chi_{B_{\gamma}^{n}(x)}(\cdot)}{p^{\gamma n}}\right)(y) & \geq \frac{1}{p^{-\operatorname{ord}(x-y) n}} \int_{B_{-\operatorname{ord}(x-y)}^{n}(x)} \frac{\chi_{B_{\gamma}^{n}(x)}(z)}{p^{\gamma n}} d z \\
& \geq \frac{\chi_{\mathbb{Q}_{p}^{n} \backslash B_{\gamma}^{n}(x)}(y)}{\|x-y\|_{p}^{n}} .
\end{aligned}
$$

From the pointwise estimate (58), the boundedness of the maximal operator $M$ (see (23)), and (46), we see that

$$
\begin{aligned}
\left\|\frac{\chi_{\mathbb{Q}_{p}^{n} \backslash B_{\gamma}^{n}(x)}(\cdot)}{\|x-\cdot\|_{p}^{n}}\right\|_{L^{q(\cdot)}\left(\mathbb{Q}_{p}^{n}\right)} & \leq\left\|M\left(\frac{\chi_{B_{\gamma}^{n}(x)}(\cdot)}{p^{\gamma^{n}}}\right)(y)\right\|_{L^{q(\cdot)}\left(\mathbb{Q}_{p}^{n}\right)} \\
& \leq C \frac{1}{p^{\gamma n}}\left\|\chi_{B_{\gamma}^{n}(x)}(\cdot)\right\|_{L^{q(\cdot)}\left(\mathbb{Q}_{p}^{n}\right)} \leq C p^{\gamma n / q(x, \gamma)-\gamma n},
\end{aligned}
$$

which proves the estimate for $\beta=n$. The case for general $\beta$ follows from the case $\beta=n$ and the identity $\left\||f|^{\beta}\right\|_{L^{q(\cdot)}\left(\mathbb{Q}_{p}^{n}\right)}$ $=\left\||f|^{n}\right\|_{L^{\beta q(\cdot) / n}\left(\mathbb{Q}_{p}^{n}\right)}^{\beta / n}$.

Lemma 9. Let $f \in L^{q(\cdot)}\left(\Omega_{p}^{n}\right)$ with $\|f\|_{L^{q(\cdot)}\left(\Omega_{p}^{n}\right)} \leq 1$ and $q \in W_{0}$ $\left(\Omega_{p}^{n}\right)$, where $\Omega_{p}^{n}$ is a bounded open subset of $\mathbb{Q}_{p}^{n}$. Then,

$$
I_{\alpha} f(x) \leq C(M f(x))^{q(x) / q^{\#}(x)}, \quad x \in \Omega_{p}^{n},
$$


where $q^{\#}$ is the Sobolev limiting exponent defined by (31) and the constant does not depend on $f$ and $x$.

Proof. Assuming $f(x)=0$ for $x \in \mathbb{Q}_{p}^{n} \backslash \Omega_{p}^{n}$, we have

$$
\begin{aligned}
\left|\int_{\Omega_{p}^{n}} \frac{f(y)}{\|x-y\|_{p}^{n-\alpha}} d y\right| \leq & \int_{B_{\gamma}^{n}(x)} \frac{|f(y)|}{\|x-y\|_{p}^{n-\alpha}} d y \\
& +\int_{\mathbb{Q}_{p}^{n} \backslash B_{\gamma}^{n}(x)} \frac{|f(y)|}{\|x-y\|_{p}^{n-\alpha}} d y \lesssim p^{\gamma \alpha} M f(x) \\
& +\int_{\mathbb{Q}_{p}^{n} \backslash B_{\gamma}^{n}(x)} \frac{|f(y)|}{\|x-y\|_{p}^{n-\alpha}} d y,
\end{aligned}
$$

where the estimate in the second inequality follows taking $\varepsilon=\alpha$ in (36) (we are indeed allowed to take $\varepsilon=$ $\alpha$ in that estimate).

To estimate the integral over the exterior of the ball, using the extension $\breve{q}$ exponent (30), Hölder's inequality, and the estimate (57), we have

$$
\begin{aligned}
\int_{\mathbb{Q}_{p}^{n} \backslash B_{\gamma}^{n}(x)} \frac{|f(y)|}{\|x-y\|_{p}^{n-\alpha}} d y & \lesssim\|f\|_{L^{q(\cdot)}\left(\Omega_{p}^{n}\right)}\left\|\frac{\chi_{\mathbb{Q}_{p}^{n} \backslash B_{\gamma}^{n}(x)}(\cdot)}{\|x-\cdot\|_{p}^{n-\alpha}}\right\|_{L^{(\bar{q})^{\prime}(\cdot)}\left(\mathbb{Q}_{p}^{n}\right)} \\
& \lesssim p^{\gamma n / q^{\prime}(x)-\gamma n+\gamma \alpha},
\end{aligned}
$$

where, in the last inequality, we use the fact that $\breve{q}(\infty)$ $\geq q(x)$ for $x \in \Omega_{p}^{n}$. From the previous estimates, when $\|f\|_{L^{q(\cdot)}\left(\Omega_{p}^{n}\right)} \leq 1$, we have

$$
\left|I^{\alpha} f(x)\right| \lesssim p^{\gamma \alpha} M f(x)+p^{\gamma \alpha-\gamma n / q(x)}
$$

Choosing

$$
\gamma=\left\lceil-\log _{p}\left(\left(M f(x)^{q(x) / n}\right)\right)\right\rceil
$$

and replacing it in (63), we obtain (60).

Theorem 10. Let $q \in W_{0}\left(\Omega_{p}^{n}\right)$ with $1 \leq q^{-} \leq q^{+}<\alpha / n$ and $\Omega_{p}^{n}$ be a bounded open set in $\mathbb{Q}_{p}^{n}$. Then, the operator

$$
I_{\alpha}: L^{q(\cdot)}\left(\Omega_{p}^{n}\right) \longrightarrow L^{q^{\#}(\cdot)}\left(\Omega_{p}^{n}\right)
$$

is bounded, where $q^{\#}$ is defined by (31).
Proof. Let us take $\|f\|_{L^{q(\cdot)}\left(\Omega_{p}^{n}\right)} \leq 1$. From (60), (18), and the boundedness of the maximal operator (24), we have

$$
\begin{aligned}
\int_{\Omega_{p}^{n}}\left|I_{\alpha} f(x)\right|^{q^{\#}(x)} d x & \leq \int_{\Omega_{p}^{n}}|M f(x)|^{q(x)} d x \\
& \leq\left(1+\|M f\|_{L^{q(\cdot)}\left(\Omega_{p}^{n}\right)}\right)^{q^{+}} \lesssim\left(1+\|f\|_{L^{q(\cdot)}\left(\Omega_{p}^{n}\right)}\right)^{q^{+}} \leqslant 1 .
\end{aligned}
$$

The result now follows from homogeneity of the norm.

\section{Applications to $p$-adic Derivative}

When we work with functions of a $p$-adic variable, $f: \mathbb{Q}_{p}^{n}$ $\longrightarrow \mathbb{C}$, it is not possible to define the derivative in the classical sense (as a limit); therefore, we must resort to the pseudodifferential operators to supply such need. The most popular operator in the $p$-adic numbers that plays the role of the derivative is the Taibleson operator (not local operator).

First, we need the Fourier transform. We set $\chi_{p}(y)=$ $\exp \left(2 \pi i\{y\}_{p}\right)$ for $y \in \mathbb{Q}_{p}$. The map $\chi_{p}(\cdot)$ is an additive character on $\mathbb{Q}_{p}$, i.e., a continuous map from $\mathbb{Q}_{p}$ into the unit circle satisfying $\chi_{p}\left(y_{0}+y_{1}\right)=\chi_{p}\left(y_{0}\right) \chi_{p}\left(y_{1}\right), y_{0}, y_{1} \in \mathbb{Q}_{p}$.

Given $\xi=\left(\xi_{1}, \cdots, \xi_{n}\right)$ and $x=\left(x_{1}, \cdots, x_{n}\right) \in \mathbb{Q}_{p}^{n}$, we set $\xi$ $\cdot x:=\sum_{j=1}^{n} \xi_{j} x_{j}$. The Fourier transform of $\varphi \in S\left(\mathbb{Q}_{p}^{n}\right)$ is defined as

$$
(\mathscr{F} \varphi)(\xi)=\int_{\mathbb{Q}_{p}^{n}} \chi_{p}(-\xi \cdot x) \varphi(x) d x \quad \text { for } \xi \in \mathbb{Q}_{p}^{n},
$$

where $d x=d^{n} x$ is the Haar measure on $\mathbb{Q}_{p}^{n}$ normalized by the condition $\operatorname{Vol}\left(B_{0}^{n}\right)=1$.

The Fourier transform is a linear isomorphism from $S\left(\mathbb{Q}_{p}^{n}\right)$ onto itself satisfying $(\mathscr{F}(\mathscr{F} \varphi))(\xi)=\varphi(-\xi)$. We will also use the notation $\mathscr{F}_{x \longrightarrow \xi} \varphi$ and $\widehat{\varphi}$ for the Fourier transform of $\varphi$.

Definition 11. The Taibleson pseudodifferential is given by operator

$$
\left(D_{T}^{\alpha} \varphi\right)(x)=\mathscr{F}^{-1}\left(\|\xi\|_{p}^{\alpha} \mathscr{F} \varphi\right), \quad \varphi \in S\left(\mathbb{Q}_{p}^{n}\right), \alpha>0 .
$$

This operator can be expressed in the following way:

$\left(D_{T}^{\alpha} \varphi\right)(x)=\left(R_{-\alpha} * \varphi\right)(x)=\frac{1-p^{\alpha}}{1-p^{-\alpha-n}} \int_{\mathbb{Q}_{p}^{n}} \frac{\varphi(x-y)-\varphi(x)}{\|y\|_{p}^{n+\alpha}} \mathrm{d} y$,

where $R_{\alpha}$ is the $p$-adic Riesz kernel; see, e.g., [25]. The right-hand side of the last equation makes sense for a wider class of functions, for example, for locally constant functions $\varphi(x)$ satisfying 


$$
\int_{\|x\|_{p} \geq 1} \frac{|\varphi(x)|}{\|x\|_{p}^{n+\alpha}} \mathrm{d} x<\infty
$$

Remark 12. We note that the constant functions $\varphi \in \operatorname{Dom}\left(D_{T}^{\alpha}\right)$ ; furthermore, $D_{T}^{\alpha} \varphi=0$. Unfortunately, the operator $D_{T}^{\alpha}$ does not satisfy the Leibniz rule for derivative of a product.

The operator $D_{T}^{\alpha}$ given by Definition 11 can be extended to the maximal domain $\left\{\varphi \in L^{2}\left(\mathbb{Q}_{p}^{n}\right):\|\xi\|_{p}^{\alpha} \mathscr{F} \varphi(\xi) \in L^{2}\left(\mathbb{Q}_{p}^{n}\right)\right\}$; thus, this operator is not bounded, and also, its spectrum is $\left\{p^{j}\right\}_{j \in \mathbb{Z}} \cup\{0\}$.

Theorem 13. Let $-n<\alpha<0,1<q^{-} \leq q^{+} \leq n /|\alpha|$, and $q \in$ $W_{0}^{\infty}\left(\mathbb{Q}_{p}^{n}\right)$. Then, the Taibleson operator

$$
D_{T}^{\alpha}: L^{q(\cdot)}\left(\mathbb{Q}_{p}^{n}\right) \longrightarrow L^{q^{\#}(\cdot)}\left(\mathbb{Q}_{p}^{n}\right)
$$

is well-defined and bounded, where $1 / q^{\#}=1 / q(x)+\alpha / n$. If $q$ $\in W_{0}\left(\Omega_{p}^{n}\right)$, then $D_{T}^{\alpha}: L^{q(\cdot)}\left(\Omega_{p}^{n}\right) \longrightarrow L^{q^{\#}(\cdot)}\left(\Omega_{p}^{n}\right)$ is bounded.

Proof. Note that, under the hypothesis of the theorem, we have $D_{T}^{\alpha} f(x)=I_{-\alpha} f(x)$. The result now follows from Theorem 6 and Theorem 10 .

In what follows, we are interested to study the following semilinear Cauchy problem $(\alpha<0)$ :

$$
\left\{\begin{array}{l}
\frac{\partial u(x, t)}{\partial t}+D_{T}^{\alpha} u(x, t)=f(u(x, t)) \\
u(x, 0)=u_{0}(x)
\end{array}\right.
$$

where $f: \mathbb{R} \longrightarrow \mathbb{R}$ is a Lipschitz function.

Lemma 14. Let $-n<\alpha<0,1<q^{-} \leq q^{+}<n /|\alpha|, q \in W_{0}\left(\Omega_{p}^{n}\right)$, where $\left|\Omega_{p}^{n}\right|<\infty$. Then, the operator $\operatorname{Tu}(x, t):=-D_{T}^{\alpha} u(x, t)$ $+f(u(x, t))$ is Lipschitz, i.e.,

$$
\|T u(x, t)-T v(x, t)\|_{L^{q(\cdot)}\left(\Omega_{p}^{n}\right)} \leq C\|u(x, t)-v(x, t)\|_{L^{q(\cdot)}\left(\Omega_{p}^{n}\right)} .
$$

Proof. From the embedding $L^{q^{\#}(\cdot)}\left(\Omega_{p}^{n}\right) \hookrightarrow L^{q(\cdot)}\left(\Omega_{p}^{n}\right)$ and Theorem 10, we see that

$$
\begin{aligned}
\|T u(x, t)-T v(x, t)\|_{L^{q(\cdot)}\left(\Omega_{p}^{n}\right)} \leq\|T u(x, t)-T v(x, t)\|_{L^{q^{\#}(\cdot)}\left(\Omega_{p}^{n}\right)} \\
\quad+\|f(u(x, t))-f(v(x, t))\|_{L^{q(\cdot)}\left(\Omega_{p}^{n}\right)} \\
\leq\left\|D_{T}^{\alpha}(u-v)(x, t)\right\|_{q^{\#}(\cdot)}+C_{1}\|u(x, t)-v(x, t)\|_{L^{q(\cdot)}\left(\Omega_{p}^{n}\right)} \\
\leq C\|u(x, t)-v(x, t)\|_{L^{q(\cdot)}\left(\Omega_{p}^{n}\right)},
\end{aligned}
$$

which ends the proof.
Theorem 15. Let $-n<\alpha<0, \quad 1<q^{-} \leq q^{+}<n /|\alpha|, \quad q \epsilon$ $W_{0}\left(\Omega_{p}^{n}\right)$, where $\left|\Omega_{p}^{n}\right|<\infty$. Then, the Cauchy problem (72) has a unique solution in $L^{q(\cdot)}\left(\mathbb{Q}_{p}^{n}\right)$.

Proof. This follows immediately from the Cauchy-LipschitzPicard theorem.

\section{Data Availability}

No data were used to support this study.

\section{Conflicts of Interest}

The authors declare that there is no conflict of interest regarding the publication of this paper.

\section{Authors' Contributions}

All authors contributed equally to this work. And all authors typed, read, and approved the final manuscript.

\section{Acknowledgments}

L.F. Chacón-Cortés was partially supported by the research project with ID-PPTA: 00009123, of the Faculty of Sciences of the Pontificia Universidad Javeriana, Bogotá, Colombia. H. Rafeiro was supported by a Research StartUp Grant of the United Arab Emirates University via Grant No. G00002994.

\section{References}

[1] V. A. Avetisov, A. K. Bikulov, and V. A. Osipov, " $p$-adic models of ultrametric diffusion in the conformational dynamics of macromolecules," Proceedings of the Steklov Institute of Mathematics, vol. 245, no. 2, pp. 48-57, 2004.

[2] V. A. Avetisov, A. K. Bikulov, and V. A. Osipov, "p-adic description of characteristic relaxation in complex systems," Journal of Physics A: Mathematical and General, vol. 35, no. 15, pp. 4239-4246, 2003.

[3] B. Dragovich, A. Y. Khrennikov, S. V. Kozyrev, and I. V. Volovich, "On p-adic mathematical physics," p-Adic Numbers, Ultrametric Analysis, and Applications, vol. 1, no. 1, pp. 1-17, 2009.

[4] R. Rammal, G. Toulouse, and M. A. Virasoro, "Ultrametricity for physicists," Review of Modern Physics, vol. 58, no. 3, pp. 765-788, 1986.

[5] W. Orlicz, "Über konjugierte exponentenfolgen," Studia Mathematica, vol. 3, no. 1, pp. 200-211, 1931.

[6] O. Kováčik and Rákosník, "On spaces $L^{q(x)}$ and $\mathrm{W}^{k, q(x) \text {," }}$ Czechoslovak Mathematical Journal, vol. 41, no. 4, pp. 592618, 1991.

[7] S. N. Antontsev and J. F. Rodrigues, "On stationary thermorheological viscous flows," Annali Dell'universita' Di Ferrara, vol. 52, no. 1, pp. 19-36, 2006.

[8] E. Acerbi and G. Mingione, "Resultats de regularite pour les fluides electrorheologiques : le cas stationnaire," Comptes Rendus Mathematique, vol. 334, no. 9, pp. 817-822, 2002. 
[9] E. Acerbi and G. Mingione, "Regularity results for stationary electro-rheological fluids," Archive for Rational Mechanics and Analysis, vol. 164, no. 3, pp. 213-259, 2002.

[10] M. Ružička, "Electrorheological fluids, modeling and mathematical theory," in Lecture Notes in Mathematics, SpringerVerlag, Berlin, 2000.

[11] M. Ružička, "Modeling, mathematical and numerical analysis of electrorheological fluids," Applications of Mathematics, vol. 49, no. 6, pp. 565-609, 2004.

[12] P. Harjulehto, P. Hästö, Ú. V. Lê, and M. Nuortio, "Overview of differential equations with non-standard growth," Nonlinear Analysis. Theory, Methods \& Applications. An International Multidisciplinary Journal, vol. 72, no. 12, pp. 4551-4574, 2010.

[13] G. Mingione, "Regularity of minima: an invitation to the dark side of the calculus of variations," Applications of Mathematics, vol. 51, no. 4, pp. 355-426, 2006.

[14] R. Aboulaich, S. Boujena, and E. El Guarmah, "Sur un modèle non-linéaire pour le débruitage de l'image," in Comptes Rendus Mathématique, vol. 345, no. 8pp. 425-429, Académie des Sciences, Paris, 2007.

[15] R. Aboulaich, D. Meskine, and A. Souissi, "New diffusion models in image processing," Computers \& Mathematics with Applications, vol. 56, no. 4, pp. 874-882, 2008.

[16] P. Blomgren, T. Chan, P. Mulet, and C. K. Wong, "Total variation image restoration, numerical methods and extensions," in Proceedings of the 1997 IEEE International Conference on Image Processing, vol. 3, pp. 384-387, Santa Barbara, CA, USA, 1997.

[17] E. M. Bollt, R. Chartrand, S. Esedoglu, P. Schultz, and K. R. Vixie, "Graduated adaptive image denoising: local compromise between total variation and isotropic diffusion," Advances in Computational Mathematics, vol. 31, no. 1-3, pp. 61-85, 2009.

[18] Y. Chen, W. Guo, Q. Zeng, and Y. Liu, “A nonstandard smoothing in reconstruction of apparent diffusion coefficient profiles from diffusion weighted images," Inverse Problems and Imaging, vol. 2, no. 2, pp. 205-224, 2008.

[19] Y. Chen, S. Levine, and M. Rao, "Variable exponent, linear growth functionals in image restoration," SIAM Journal on Applied Mathematics, vol. 66, no. 4, pp. 1383-1406, 2006.

[20] T. Wunderli, "On time flows of minimizers of general convex functionals of linear growth with variable exponent in BV space and stability of pseudo-solutions," Journal of Mathematical Analysis and Applications, vol. 364, no. 2, pp. 591-598, 2010.

[21] N. M. Chuong and H. D. Dung, "Maximal functions and weighted norm inequalities on local fields," Applied and Computational Harmonic Analysis, vol. 29, no. 3, pp. 272-286, 2010.

[22] D. Cruz-Uribe and A. Fiorenza, Variable Lebesgue Spaces: Foundations and Harmonic Analysis, Birkhäuser, 2013.

[23] L. Diening, P. Harjulehto, P. Hästö, and M. Ružička, "Lebesgue and Sobolev spaces with variable exponents," in Lecture Notes in Mathematics, Springer-Verlag, Berlin, 2011.

[24] H. Rafeiro and E. Rojas, Espacios de Lebesgue con exponente variable: un espacio de Banach de funciones medibles (Spanish), IVIC-Instituto Venezolano de Investigaciones Científicas, 2014.

[25] M. H. Taibleson, Fourier Analysis on Local Fields, Princeton University Press, 1975.
[26] L. F. Chacón-Cortés and H. Rafeiro, "Variable exponent Lebesgue spaces and Hardy-Littlewood maximal function on p-adic numbers," p-Adic Numbers, Ultrametric Analysis, and Applications, vol. 12, no. 2, pp. 90-111, 2020.

[27] C. Capone, D. Cruz-Uribe, and A. Fiorenza, "The fractional maximal operator and fractional integrals on variable $L^{p}$ spaces," Revista Matemática Iberoamericana, vol. 23, no. 3, pp. 743-770, 2007.

[28] V. S. Vladimirov, I. V. Volovich, and E. I. Zelenov, p-adic Analysis and Mathematical Physics, World Scientific, 1994.

[29] E. J. McShane, "Extension of range of functions," Bulletin American Mathematical Society, vol. 40, no. 12, pp. 837-843, 1934.

[30] S. Cobzas, R. Miculescu, and A. Nicolae, Lipschitz Functions, Lecture Notes in Mathematics, Springer-Verlag, Berlin, 2011.

[31] G. V. Welland, "Weighted norm inequalities for fractional integrals," Proceedings of the American Mathematical Society, vol. 51, pp. 143-148, 1975. 